\title{
A Review On Lyme Disease
}

\author{
Bandari Vamshi Krishna ${ }^{1}$, Thogaru Sandeep Reddy ${ }^{1}$, K. Rohith Kumar* \\ 1.Department of Pharmacy Practice, Sree Chaitanya institute of Pharmaceutical Sciences
}

\section{ABSTRACT}

Lyme borreliosis, also known as Lyme disease, is a multi-organ animal-borne disease, caused by bacteria - spirochetes of the Borrelia species classified as Borrelia burgdorferi $(\mathrm{Bb})$ strain (sensu lato). It is the most common tick-borne infectious disease in Europe and USA. The infection is transmitted by ticks of the species Ixodes ricinus. . In Europe, I. ricinus ticks usually prey on rodents and deer, Humans are infected through a tick bite to the skin. Bb has to be attached for at least $24 \mathrm{~h}$ for an infection to result. The risk of infection increases with length of time of human exposure to the tick, approaching $100 \%$ on the third day. Thus, early removal of ticks is the best method of Lyme borreliosis prophylaxis. The most common manifestation of Lyme disease, erythema migrans, appears at the site of a tick bite 3-30 days (but typically within 7-10 days) after the bite. It is recognized in more than $90 \%$ of patients who have objective evidence of $B$. burgdorferi infection. Erythema migrans is usually asymptomatic but may be pruritic or painful, and it may be accompanied by systemic clinical features such as fever, malaise, headache, myalgia, or arthralgia. A two-step diagnosis is necessary: the first step is based on a high sensitivity ELISA test with positive results confirmed by a more specific Western blot assay. Antibiotic therapy is curative in most cases, but some patients develop chronic symptoms, which do not respond to antibiotics.

Keywords: Lyme borreliosis ; ELISA; tick-borne; Western blot assay 


\section{INTRODUCTION}

Lyme borreliosis, also known as Lyme disease, is a multi-organ animal-borne disease, caused by bacteria - spirochetes of the Borrelia species classified as Borrelia burgdorferi $(\mathrm{Bb})$ strain (sensu lato). It is the most common tick-borne infectious disease in Europe. The infections transmitted by ticks of the species Ixodes ricinus. Spirochetes have also been isolated from mosquitoes, fleas and flies, but they were found to play no role in the transmission of the infection to humans. In Europe, I. ricinus ticks usually prey on rodents and deer, which are the key $\mathrm{Bb}$ reservoir ${ }^{[1-4]}$.At least $11 \mathrm{Bb}$ genostrains are known, but not all of them are consider red pathogenic. In Europe Lyme borreliosis is caused by B. garinii, which is more likely to cause neuroborreliosis, with B. afzelii causing atrophicchronic acrodermatitis (ACA), and Bb itself (Borrelia burgdorferi sensustricto). Most recent reports suggest that in Europe, pathogenic strainsalso include B. lusitaniae, B. valaisiana and B. spielmani ${ }^{[5-7]}$. Humans are infected through a tick bite to the skin. Bb has to be attached for at least $24 \mathrm{~h}$ for an infection to result. The risk of infection increases with length of time of human exposure to the tick, approaching $100 \%$ on the third day. Thus, early removal of ticks is the best method of Lyme borreliosis prophylaxis ${ }^{[2]}$
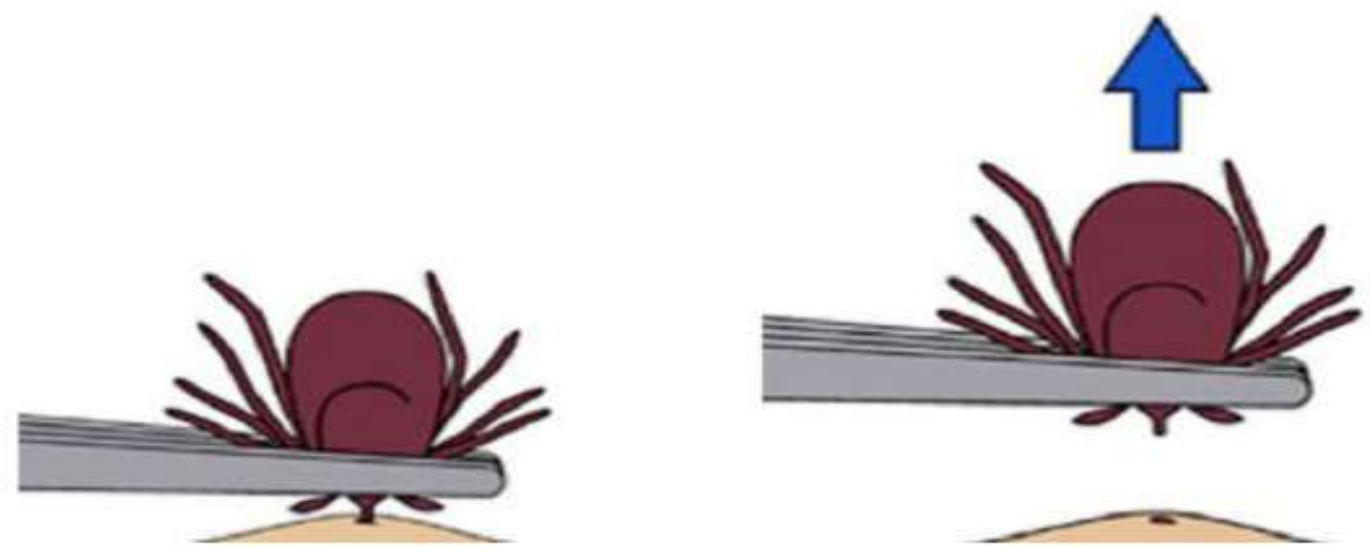

Figure 1: Pull ticks out by grasping them near the head and pulling straight up. (Centers for Disease Control and Prevention; public domain; lyme removal index)

\section{Biology of The Organism}

B. burgdorferi is a gram-negative organism that is $10-30 \mathrm{~mm}$ long and $0.2-0.25 \mathrm{~mm}$ wide. It grows best at 307C-347C in a microaerophilic atmosphere, dividing every 8-12 h during log phase growth ${ }^{[5,6]}$. It is routinely grown in liquid cultures in BSK II medium. Ultra structurally, B. burgdorferi resembles other spirochetes in the genus Borrelia, with 7-11 periplasmic flagella that are attached sub terminally to the protoplasmic cylinder and overlap in the center of the cell. 
The main structural component of the flagella is flagellin, a $41-\mathrm{kDa}$ protein. The outer membrane contains an abundance of outer surface proteins (Osps) anchored to the outer membrane via lipid moieties at their amino termini (some may also extend to the cytoplasmic membrane). These include OspA, OspB, and OspC(lipoproteins of »32, 34, and $24 \mathrm{kDa}$, respectively). Although the role these Osps play during infection is not known, different Osp genes are expressed at different times during transmission from the tick vector to the mammalian host. Another antigen of $B$. burgdorferi is the $60-\mathrm{kDa}$ antigen, which has been termed" common antigen" and belongs to the heat-shock protein family. Unlike other spirochetes, B. burgdorferi has linear chromosomes and 4-9 linear and circular plasmids. The genes that encode the major Osps of B. burgdorferi are located onplasmids. Recent phylogenetic studies based on relatedness of DNA have subdivided the organism that causes Lyme disease, B.burgdorferi sensu lato, into multiple genospecies: $B$. Burgdorferisensu stricto, Borrelia garinii, and Borrelia afzelii. In the United States, a number of genospecies of B. burgdorferi sensu latohave been isolated from animals and from ticks, but only B.burgdorferi sensu stricto has been isolated from humans. In contrast, there is substantial antigenic variability among human isolates of B. burgdorferi in Europe. ${ }^{[8]}$

\section{EPIDEMIOLOGY}

Lyme disease is the most common tick-borne disease in the United States ${ }^{[9-1]}$ Lyme disease is most commonly reported in New England and the mid-Atlantic states, upper north-central regions, and several counties in north western California. Variation in zoonotic factors, including the presence of the white-footed mouse and white tail eddeer, which are important hosts in the life cycle of the Ixodes tick, account for endemic areas in these geographic locations. In 2005, more than 23,000 cases of Lyme disease were reported to the CDC, with most occurring in New England and the Great Lakes region(Figures 1 and 2). ${ }^{[13,14]} \cdot$ In the first 5 years after the condition was described and officially named (1982-1986), the number of reported cases increased 32fold $^{[15]}$, making Lyme disease a national health concern. Since the 1980 s, the public increasingly has become aware of the harmful effects of untreated Lymedisease. Because the condition is commonly discussed on Internet sites and in the media, the concerns about its complications and prevalence have, in some cases, been regarded as out of proportion to the actual threat of disease. In 2001, Leonard H. Sigal, MD, was quoted by the New York Times as explaining that "Lyme disease, although a problem, is not nearly as big a problem as most people think. The bigger epidemic is Lyme anxiety."[16] Although Lyme disease may not be a problem in many parts of the United States, the disease is a considerable threat to residents of endemic areas. Even President George W. Bush recently received treatment for Lyme disease. The age distribution of Lyme 
disease is bimodal, with the highest number of cases occurring in children aged 5 to 14 years and adults aged 55 to 70 years $^{[13]}$. Those who reside or are employed in areas where ticks are commonly found are at increased risk. Outdoor activities such as hiking, camping, hunting, fishing, and gardening place people at risk, as does working around heavy brush or wooded areas. However, most infections occur in residential areas during routine activities. Lyme disease remains a major focus in public health. The Healthy People 2010 public health goals developed by the US Department of Health and Human Services aimed for a $44 \%$ decrease in the incidence of Lyme disease by2010 ${ }^{[17]}$. Currently, the CDC awards more than $\$ 3.5$ million per year for new research on Lyme disease ${ }^{[18]}$. This review details the clinical presentation of the disease as well as risk factors, treatment, and prophylaxis. To obtain in formation, we used the keyword Lyme disease to search the PubMed and MEDLINE databases for articles published from August 31, 1997, through September 1, 2007.

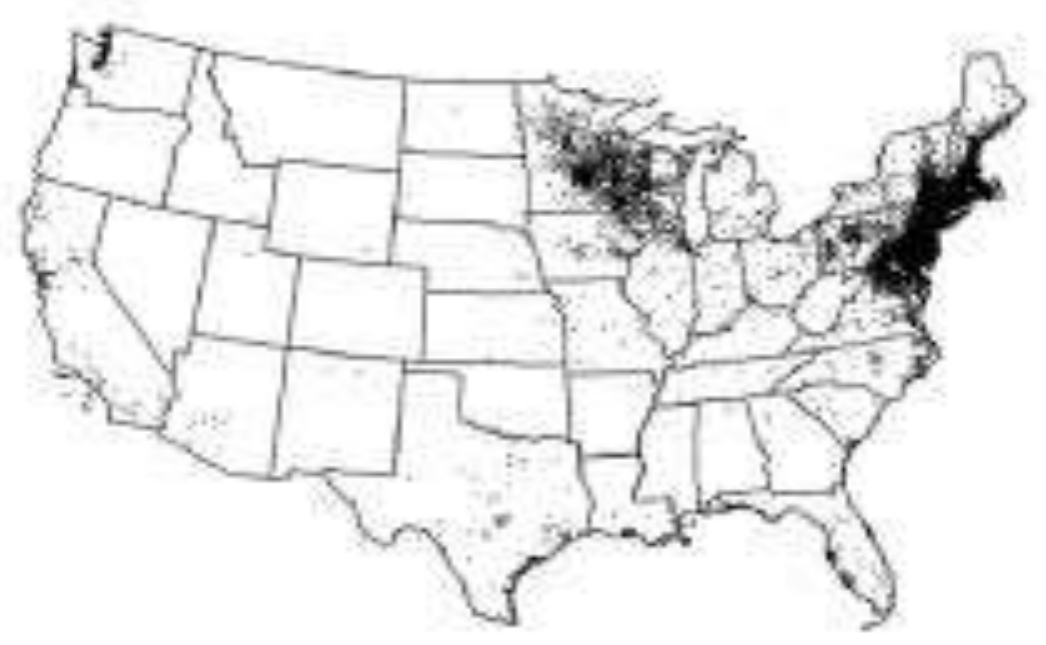

Figure 1. Reported cases of Lyme disease in the United States in2005. Most cases were reported in New England (Connecticut, Rhode Island, New York, Pennsylvania, Delaware, New Jersey, Maryland, and Massachusetts) and in the Great Lakes region (Wisconsin and Minnesota). One dot is placed randomly within the county of residence for each reported case. From the Centers for Disease Control and Prevention. ${ }^{[13]}$. ETIOLOGY AND VECTOR LIFE CYCLE

The white-footed mouse is the primary animal reservoir for Lyme disease in the United States. Black-legged ticks (Iscapularis, deer ticks) are responsible for transmitting Lyme disease bacteria (B burgdorferi) to humans in the New England and Great Lakes areas. In the West, Bburgdorferi is transmitted to humans by the western blacklegged tick (Ixodes pacificus), 
although the incidence of the bacteria in these ticks is much lower. The nymphal and larval forms of the Ixodes tick feed primarily on the white-footed mouse, whereas the adult ticks are found on deer (Figure 3). Ixodes ticks are much smaller than common dog ticks, only a few millimeters in diameter in the larval and nymphal stages. The nymph form of the deer tick, often implicated in bites, is smaller than the adult form. Ixodes ticks are most likely to transmit infection after feeding for at least 36 to 48 hours; however, the minimum time may be as little as 24 hours. The infection is usually noted from April to November ${ }^{[13]}$. Other mammals, including dogs, can be hosts and can develop Lyme disease, but transmission from dogs to humans does not occur. Fleas, flies, and mosquitoes are not vectors for Lyme disease.

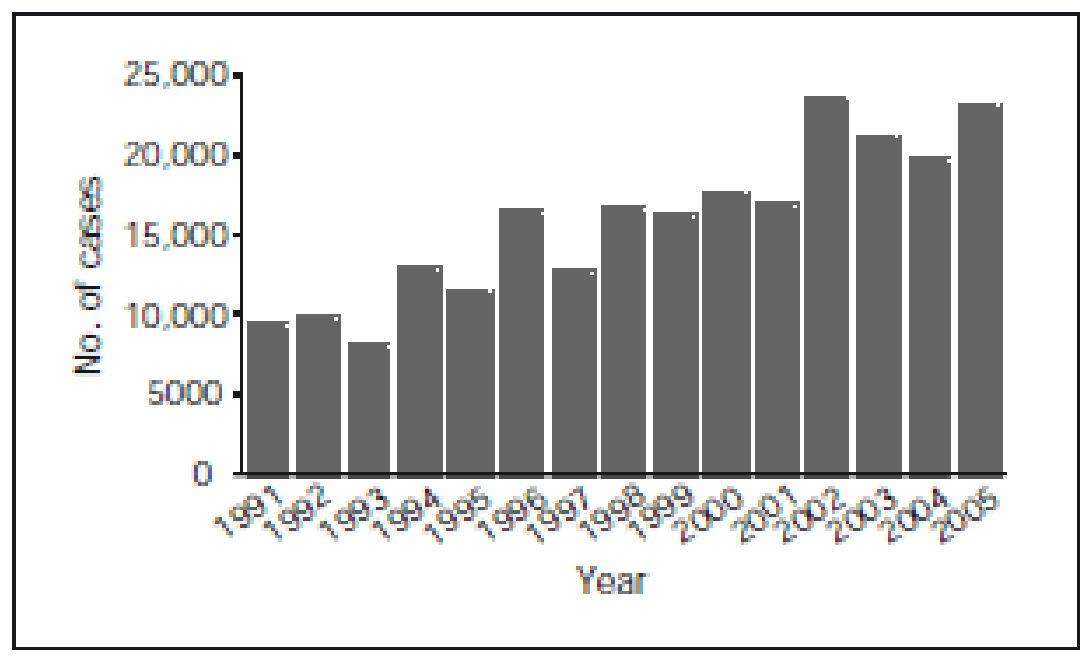

Figure 2: Reported cases of Lyme disease in the United States by year, 1991-2005. From the Centers for Disease Control and Prevention ${ }^{[13]}$.

\section{CLINICAL MANIFESTATIONS}

The clinical manifestations of Lyme disease depend on the stage of the illness-early localized disease; early disseminated disease; or late disease ${ }^{[19,20]}$. The most common manifestation of early localized Lyme disease, erythema migrans (figure3 and 4), appears at the site of a tick bite 3-30 days (but typically within 7-10 days) after the bite. It is recognized in $\gg 90 \%$ of patients who have objective evidence of $B$. burgdorferi infection ${ }^{[20,21]}$. Erythema migrans begins as a red macule or papule and, if untreated, expands for days to weeks to form a large, annular, erythematous lesion that is as much as $70 \mathrm{~cm}$ in diameter(median, $15 \mathrm{~cm}$ ). This rash may be uniformly erythematous or it may appear as a target lesion with variable degrees of central clearing. It can vary greatly in shape and occasionally may have vesicular or necrotic areas in the center. Erythema migrans is usually asymptomatic but may be pruritic or painful, and it may be accompanied by systemic clinical features such as fever, malaise, headache, myalgia, or 
arthralgia. Other clinical findings that may be associated with erythema migrans include headache, regional lymphadenopathy, and a stiff neck. Conjunctivitis has also been described in patients with erythema migrans, but in recent studies of culture-positive patients with erythema migrans, conjunctivitis was rare ${ }^{[22]}$.Patients with early Lyme disease may also present with an illness similar to those caused by viruses (e.g., with fever, malaise, and myalgia) without erythema migrans. However, because

both the sensitivity and specificity of serological tests for early Lyme disease are poor and because viral illness-like symptoms from causes other than Lyme disease are common, it is difficult to confirm a diagnosis of Lyme disease in patients with nonspecific, acute symptoms. Although the frequency of this manifestation of Lyme disease is not known, information from clinical trials of Lyme vaccine (in which subjects had routine follow-up serology done by a reference laboratory) suggests that it is uncommon. The most common manifestation of early disseminated Lyme disease is multiple erythema migrans. The secondary skin lesions, which usually appear 3-5 weeks after the tick bite, consist of multiple annular erythematous lesions similar to, but usually smaller than, the primary lesion. Other common manifestations of early disseminated Lyme disease are cranial nerve palsies, especially facial nerve palsy, and meningitis. Although rare, carditis, usually manifested by fluctuating degrees of atrioventricular block, may also occur at this stage. The carditis may be associated with dizziness, palpitations, dyspnea, chest pain or syncope. Systemic symptoms such as myalgia, arthralgia, headache, and fatigue are common in the early disseminated stage. Eye involvement (e.g., uveitis, iritis, and optic neuritis)has also been reported in this stage of Lyme disease. However, because $B$. burgdorferi has only rarely been identified in these disorders, the role of B. burgdorferi in their pathogenesis is uncertain.

The most common manifestation of late Lyme disease in the United States is arthritis, which is usually monoarticular oroligoarticular and affects the large joints, particularly the knee. The arthritis occurs weeks to months after the initial infection. Although the joint typically is swollen and tender, the intense pain associated with a septic arthritis usually is not present. About $10 \%$ of adults and fewer than $5 \%$ of children with Lymearthritis develop inflammatory joint disease that does not respond to antimicrobial agents and typically affects 1 knee for months to years. Because of the increased frequency of certainHLA-DR4 alleles in these patients, an autoimmune mechanism has been proposed ${ }^{[23-25]}$. Encephalitis, encephalopathy, and polyneuropathy are also manifestations of late Lyme disease, but these manifestations are rare ${ }^{[26,27]}$.Additional episodes of erythema migrans have been reported 
in patients who received appropriate antimicrobial therapy for an initial episode of erythema migrans. It has been assumed that prompt initiation of antimicrobial therapy for early Lyme disease can depress the humoral immune response to B. burgdorferi, thereby leaving such patients susceptible to reinfection from new infection with B. burgdorferi. However, although itis much less common in this setting, even patients with serum antibodies to B. burgdorferi after the initial episode occasionally may be susceptible to subsequent infection. The clinical manifestations of Lyme disease also may depend on which geno species of B. burgdorferi is causing the infection. The differences in geno species found in Europe and in North America may account for differences in the frequencies of certain manifestations of Lyme disease in these areas. For example, neurological manifestations of Lyme disease are more common in Europe, whereas rheumatological manifestations are more common in North America. In addition, certain skin and soft tissue manifestations of Lyme disease, such as acrodermatitischronica atrophicans and lymphocytomas, are usually caused by B. afzelii or B. garinii and, therefore, are seen in Europe but are extremely rare in the United States. In the southern United Sates, a rash that resembles erythema migrans has been associated with the bite of Amblyomma americanum (the Lone Star tick). A new presumptive species, Borrelialonestarii sp. nov., has been isolated from $A$. americanum ticks and may be the cause of this erythema migrans-like rash. Ixodes ticks may transmit several other pathogens in addition to B. burgdorferi that are capable of causing human disease, including Babesia, Ehrlichia, and viruses ${ }^{[28,29]}$. These agents may be transmitted either separately or simultaneously with B. burgdorferi. Coinfection may alter both the clinical presentation and the response to treatment of patients with Lyme disease, although the frequency with which coinfection occurs is uncertain and its impact on the clinical presentation and response to treatment is not well characterized. 


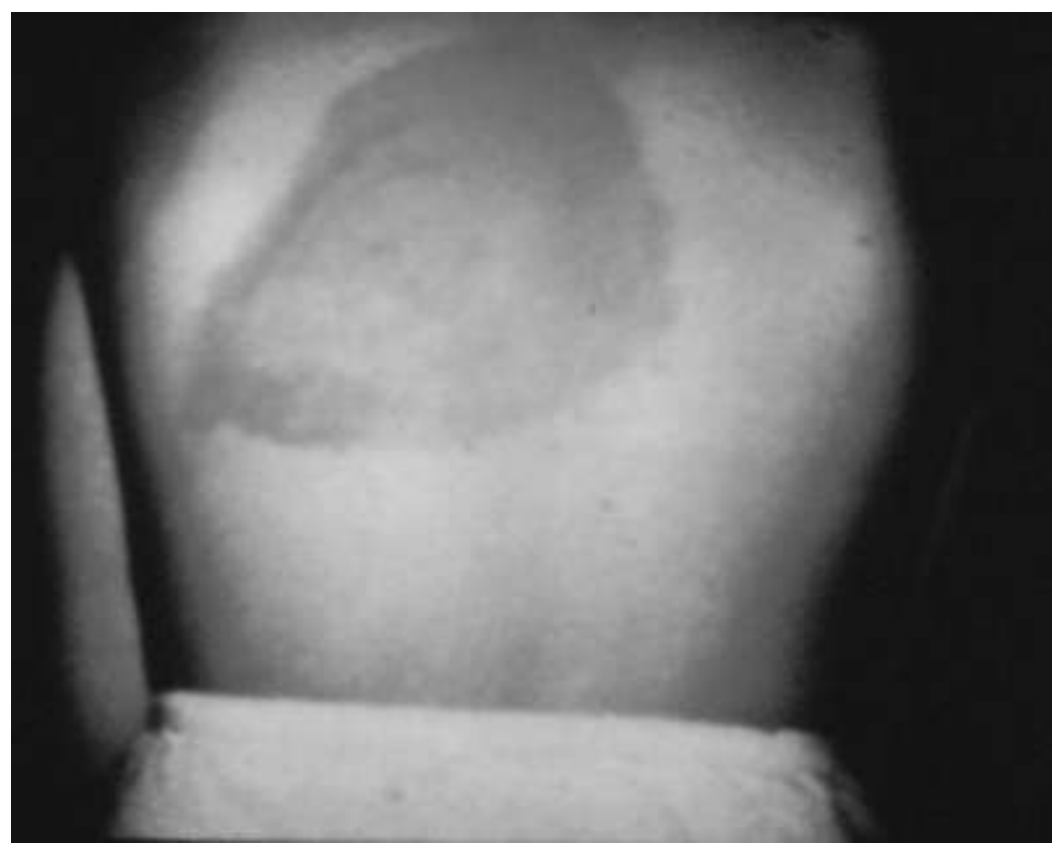

Figure 3. Erythema migrans ${ }^{[49]}$

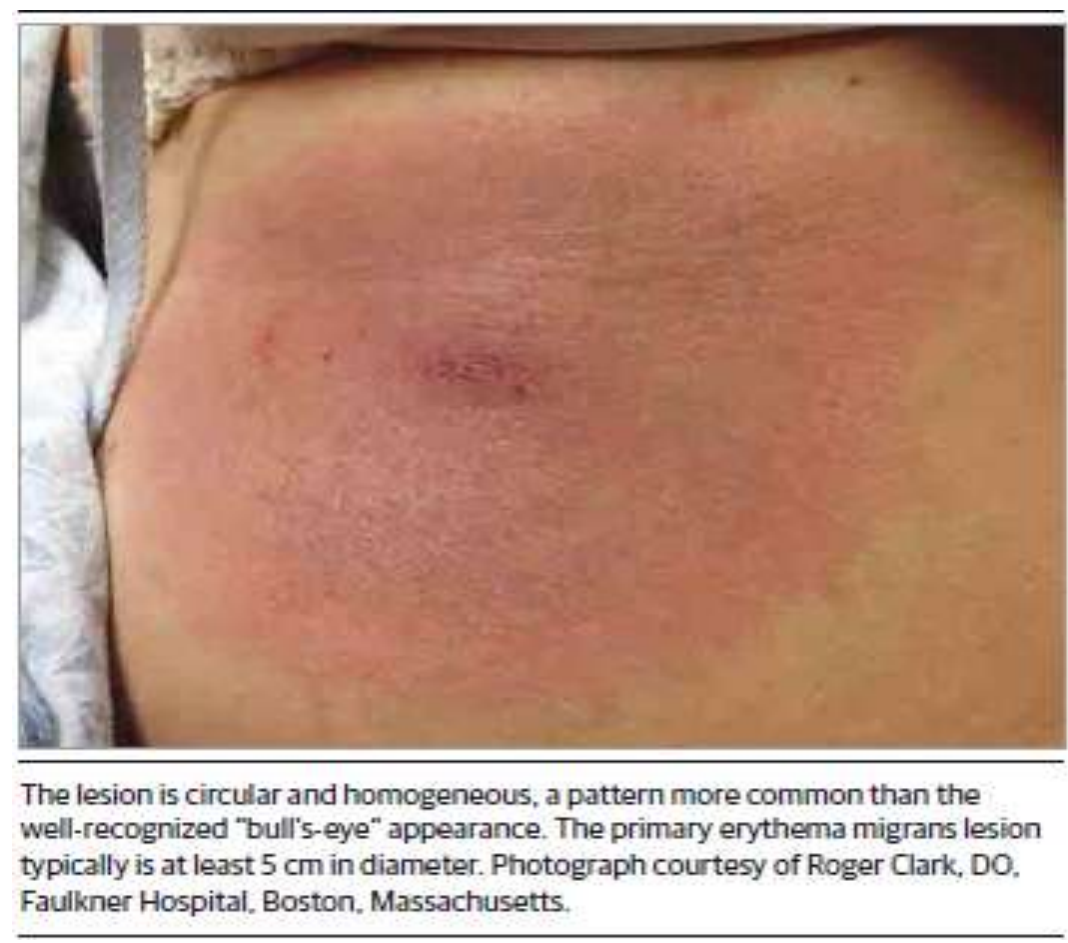

Figure 4. Erythema Migrans Skin Lesion at the Site of a Tick Biteon the Abdomen of a Patient ${ }^{[48]}$

\section{PATHOGENESIS}

Information about the pathogenesis of Lyme disease comes from both human studies and animal models. B. burgdorferi express outer surface proteins (Osps) that are important for both survivals in the tick and for infection in humans. Osp A is required for B. Burgdorferi adherence to the tick 
midgut ${ }^{[30,31]}$. Its expression decreases during engorgement as the spirochete leaves the midgut for the salivary glands and subsequent injection into the mammalian host. During this period, the expression of OspC increases; it has been postulated that OspC plays a role in migration of and infection by the spirochete ${ }^{[20]}$. The spirochete's ability to spread through skin and other tissues may be facilitated by the binding of OspC to human plasminogen ${ }^{[32]}$. This dissemination from the site of the tick bite, via the bloodstream, produces the systemic systems that may be associated with early Lyme disease as well as the clinical manifestations of early disseminated and, ultimately, of late Lyme disease. In humans with erythema migrans, infiltrates of macrophages and of $\mathrm{T}$ cells produce both inflammatory and anti-inflammatory cytokines ${ }^{[33]}$. There is also evidence that in disseminated infections, adaptive T-cell and B-cell responses in lymph nodes produce antibodies against many components of the spirochete ${ }^{[34,35]}$ During dissemination within humans, B. burgdorferi attaches to certain host integrins eliciting a pro inflammatory response, that includes production of both matrix glycosaminoglycans, and extracellular matrix proteins which may explain the organism's tropisms for particular tissues (e.g., collagen fibrils in the extracellular matrix in the heart, nervous system, and joints).

Animal models have provided information about the clinical manifestations and immune response during early, disseminated disease including neuroborreliosis and carditis. Studies in mice have clearly demonstrated the importance of inflammatory innate immune response in controlling early, disseminated Lyme disease. After infection with B. burgdorferi, mice that lack either toll-like receptor 2, a part of the innate immune system, or the toll-like receptor adaptor, MyD88, have higher bacterial loads and more severe arthritis than isogenic wild type mice. The role of complement in controlling infection has also been well described. Mice that lack C3 have increased numbers of spirochetes when infected; moreover, B.burgdoferi produces specific proteins that inhibit complement activity which facilitates infection. A $\mathrm{C} 3 \mathrm{H}$ mouse model of Lyme carditis has been developed in which cardiac infiltrates of both macrophages and $\mathrm{T}$ cells produce inflammatory cytokines. In these mice, the killing of spirochetes through cellular immune mechanisms appears to be the dominant factor in the resolution of cardiac disease.

A nonhuman, primate animal model of neuroborreliosis has been developed to try to better understand the spread of B. burgdorferi within the nervous system. autoimmune suppressed monkeys with an exceptionally large inoculum of bacteria, B. burgdorferi infiltrated the leptomeninges, the motor and sensory nerve roots, and the dorsalrootganglia, but not the brain parenchyma. B. burgdorferi also infiltrated the perineurium (the connective tissue sheath surrounding each bundle of peripheral-nerve fibers) in the peripheral nervous system of these 
monkeys. Studies of patients with clinical manifestations of late disease, specifically Lyme arthritis, have confirmed observations in animals that the host immune response is important for the pathogenesis of disease. Synovial tissue from patients with Lyme arthritis typically shows synovial hypertrophy, vascular proliferation, and a marked mononuclear cell infiltrate. Sometimes pseudo lymphoid follicles are present that resemble peripheral lymph nodes.

During acute Lyme arthritis, innate immune responses to B. burgdorferi lipoprotein, as well as marked adaptive immune responses to many spirochetal proteins, are found. BothTh-1 and Th-2 dependent cytokines are found in the joint fluid. In addition, patients with Lyme arthritis typically have higher Borrelia-specific antibody titers than do patients with other manifestation of Lyme disease. Some adult patients with Lyme arthritis, particularly those with HLA-DRB1 alleles, will develop a chronic, antibiotic treatment resistant, autoimmune arthritis[35].

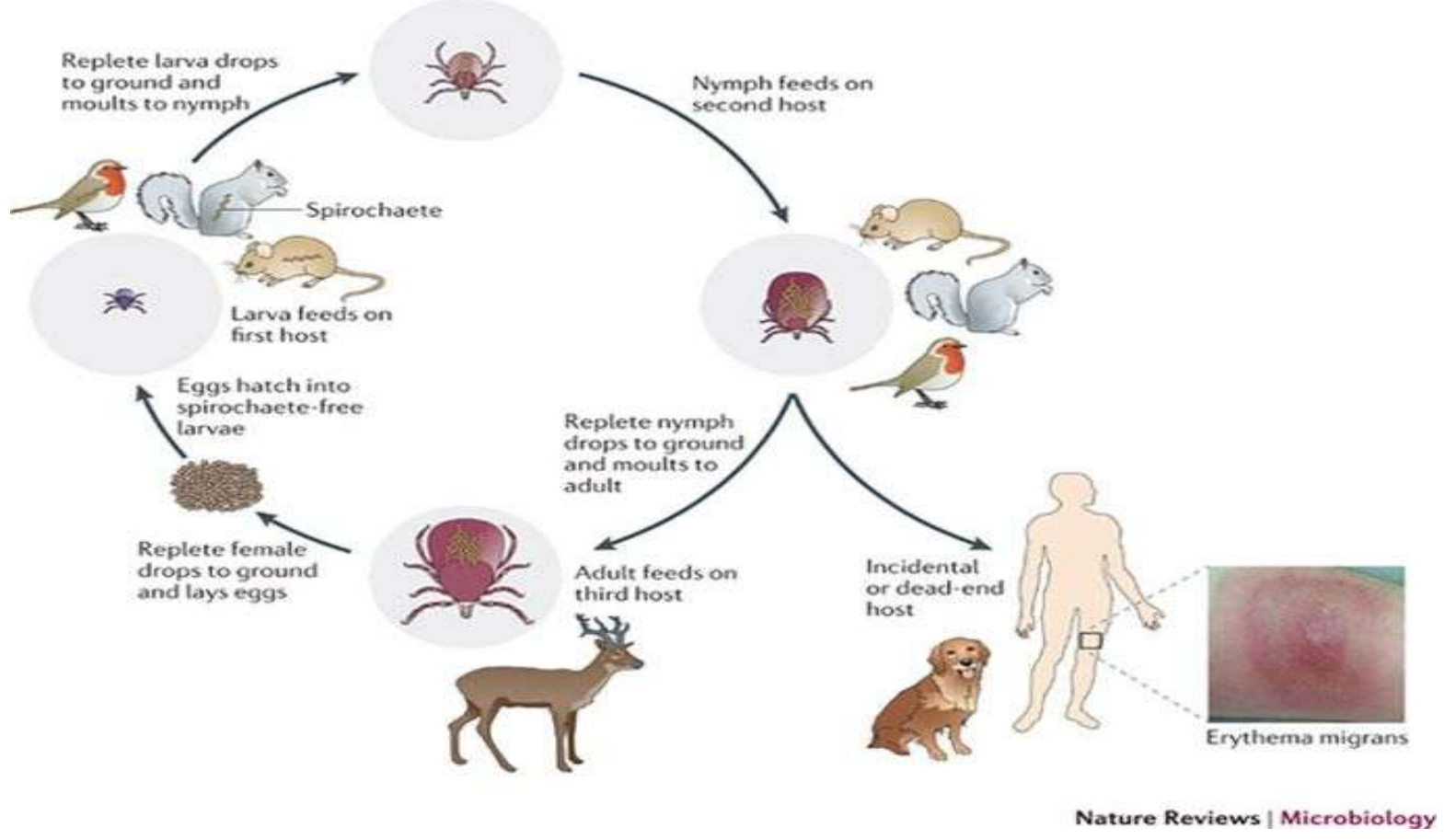

Figure: Life cycle of B. burgdorferi $(\mathrm{Bb})^{[50]}$ 
Bacteria multiply, alter gene expression, disseminate in tick

$\begin{array}{cc}\text { Bacteria are } & \text { Bacteria spread in skin: } \\ \text { inoculated into skin } & \text { erythema migrans lesion }\end{array}$
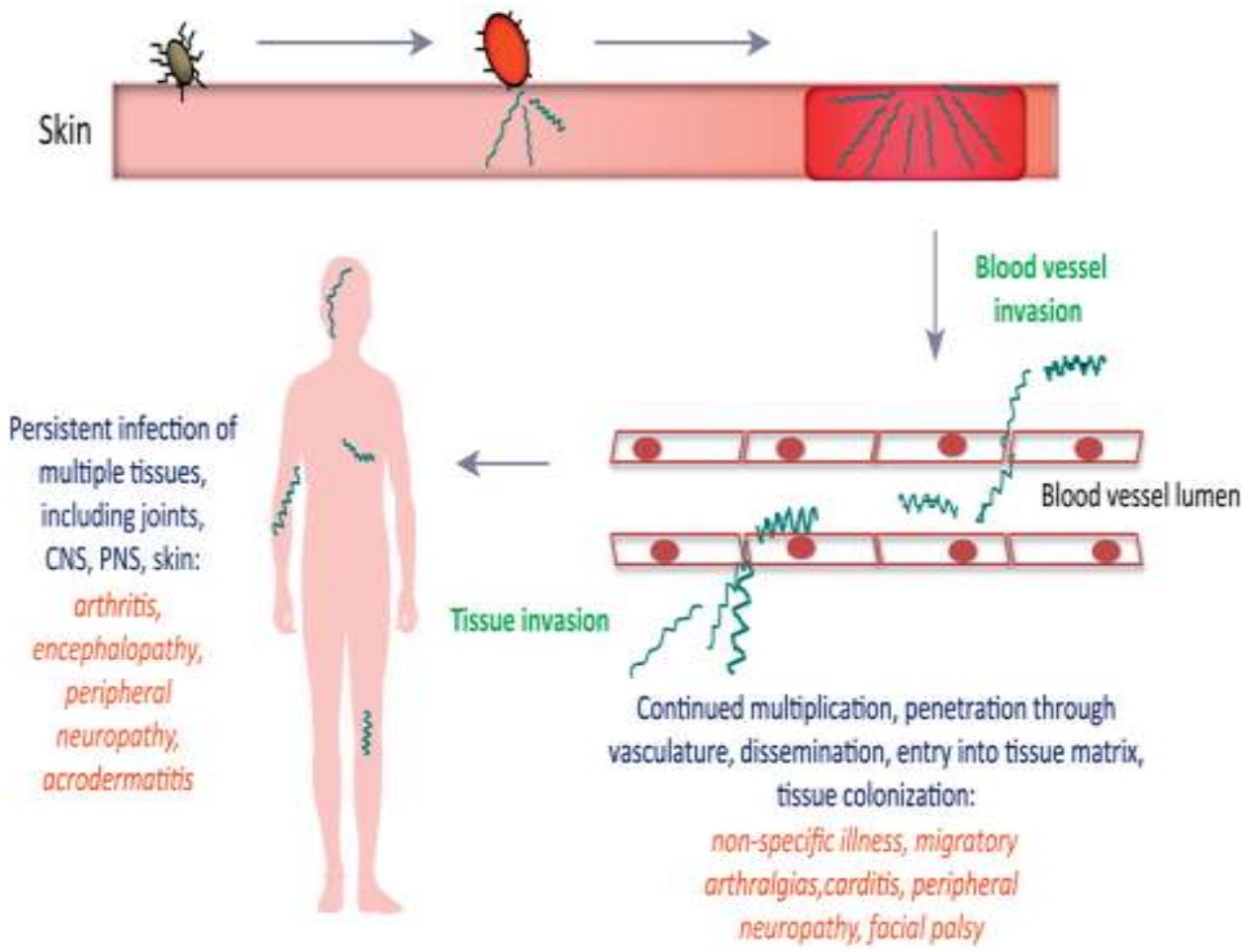

Figure: Pathogenesis of B. burgdorferi (Bb) [Lyme disease $]^{[51]}$

\section{DIAGNOSIS}

The diagnosis of Lyme disease is based on clinical features in a person who has traveled to or lives in an endemic area. In most cases, it is appropriate to treat patients who have early disease and a high pretest probability, on the basis of signs and symptoms, after a tick bite by the Ixodes species. As with other tick-borne diseases, only $50 \%$ to $70 \%$ of patients recall a tick bite ${ }^{[36]}$, often because the deer ticknymphs are small and go unnoticed. Common laboratory tests usually are not revealing in the diagnosis of Lyme disease. The white blood cell count may be elevated or normal. Hemoglobin, hematocrit, creatinine, and urinalysis results are usually normal. If neurologic involvement is present, cerebrospinal fluid samples show moderate lymphocytic pleocytosis ${ }^{[37]}$,elevated protein concentrations, and normal to slightly low glucose concentrations. Neurologic manifestations usually include headache, seventh nerve palsy (possibly bilateral),neck pain and stiffness, forgetfulness, irritability, emotional lability, and sleep disturbances. Cerebrospinal fluid pleocytosis resolves promptly with appropriate anti microbial therapy. Serologic tests for Lyme disease support but are not always essential to the diagnosis. Furthermore, the tests are prone to false-negative and false-positive results and can be misleading, especially early in the course of the disease. 
When confirmation is necessary, the CDC recommends an enzyme-linked immunosorbent assay (ELISA), which is sensitive but not necessarily specific, followed by the more specific Western immunoblot test. Western blot tests should also be ordered if an indeterminate result is obtained by ELISA or indirect fluorescent antibody (IFA) testing.

The sensitivity of the numerous ELISA and IFA tests is affected by the timing of the test. Antibodies to Bburgdorferi may not be present early in disease, potentially leading to a falsenegative result. False positives can occur with mononucleosis, autoimmune states, and Treponemapallidum infection; therefore, serologic testing is not advised when the pretest probability of Lyme disease is $\operatorname{low}(<20 \%)^{[38,39]}$. Likewise, testing is unnecessary when the pretest probability of Lyme disease exceeds $80 \%$. In this setting, empiric treatment should be initiated. In large part, the ELISA and IFA techniques share the same limitations.

The Western blot technique provides serum IgM or IgG results. Its sensitivity, like that of ELISA and IFA, depends on the timing of the test, but it is more specific. When performed at least 4 weeks after exposure, Western blot will identify most patients with Lyme disease, with fewer false-positive results than ELISA returns. Because serologic testing is not $100 \%$ sensitive or specific, some people with Lyme disease will not have confirmatory laboratory results, especially patients in endemic areas who have characteristic physical findings at presentation.

After an infection, antibodies often persist for months or years. As a result, serologic tests do not accurately distinguish active from past infection. The overall false-positive rate of Lyme disease testing is approximately $5 \%{ }^{[40]}$. Positive findings on ELISA or the IFA test followed by negative findings on Western blot usually suggest the absence of Lyme disease. This is especially true if the Western blotis performed more than 2 to 4 weeks after exposure. Positive serologic tests and a history of Lyme disease do not ensure protective immunity.

Recurrent Lyme disease has been observed in a few isolated cases. Further diagnostic options include skin biopsy of early erythema migrans lesions; B burgdorferi can be detected in $80 \%$ of cases. The culture should be taken from the perimeter of the lesion. This test is essentially $100 \%$ specific and can distinguish live from dead organisms. However, biopsies are not routinely used because of the need for both a special bacteriologic agar (modified Barbour-Stoenner-Kelly medium) and prolonged observation of cultures, both of which limit the commercial availability of culture testing ${ }^{[39]}$. Polymerase chain reaction can detect DNA of Bburgdorferi in many different types of samples, including those from skin, blood, cerebrospinal fluid, and synovial fluid. However, it is not commonly recommended for the routine diagnosis of Lyme disease ${ }^{[41]}$. 
The limited enthusiasm stems from the brief time that $B$ burgdorferi circulates in the bloodstream and the possibility of positive test results if dead spirochetes are present.

Other rapid screening tests used in the diagnosis of Lyme disease require further research before their use is recommended. The Lyme Urine Antigen Test has not been approved by the US Food and Drug Administration and is not recommended by the CDC or the American Lyme Disease Foundation. Clinicians should be aware that $15 \%$ of patients may be confected with a second tick-bornepathogen, including those causing human granulocyticana plasmosis (formerly known as human granulocyticehrlichiosis) or babesiosis. Such coinfection can alter both the clinical presentation of Lyme disease and the response to treatment ${ }^{[39]}$.

\section{TREATMENT}

Treatment of Lyme borreliosis in the phase of EM or borrelial lymphocytoma consists of doxycycline, amoxicillin or cefuroxime axetil. Arthritisis treated with oral doxycycline, amoxicillin or cefuroxime axetil, whilst neuroborreliosis, recurrent arthritis and heart involvement are treated with ceftriaxone, cefotaxime or penicillin G. The ACA is treated with amoxicillin, doxycycline, ceftriaxone, cefotaxime and penicillin G. Dosage and duration of treatment are summarized in Table $\mathrm{I}^{[42-45]}$.

Table I. Dosage and duration of Lyme disease treatment

\begin{tabular}{|c|c|c|c|c|}
\hline Clinical picture & $\begin{array}{l}\text { Suggested } \\
\text { regimens }\end{array}$ & Dosage & Administration & $\begin{array}{c}\text { Duration } \\
\text { of the treatment } \\
\text { [days] }\end{array}$ \\
\hline \multirow{3}{*}{$\begin{array}{l}E M \\
B L C\end{array}$} & Doxycycline & $100 \mathrm{mg}$ bid & po & $14-21$ \\
\hline & Amoxicillin & $500 \mathrm{mg}$ tid (children: $50 \mathrm{mg} / \mathrm{kg} /$ day) & po & $14-21$ \\
\hline & Cefuroxime & $500 \mathrm{mg}$ bid (children: $30 \mathrm{mg} / \mathrm{kg} /$ day) & po & $14-21$ \\
\hline \multirow{3}{*}{$\begin{array}{l}\text { Lyme disease } \\
\text { with joint } \\
\text { involvement }\end{array}$} & Amoxicillin & $500-1000$ mg tid (children: 50 mg/kg/day) & po & $14-28$ \\
\hline & Doxycycline & $100 \mathrm{mg}$ bid or $200 \mathrm{mg}$ q $24 \mathrm{~h}$ & po & $14-28$ \\
\hline & Cefuroxime & $500 \mathrm{mg}$ bid (children: $15 \mathrm{mg} / \mathrm{kg} /$ day) & po & $14-28$ \\
\hline \multirow{3}{*}{$\begin{array}{l}\text { Lyme disease } \\
\text { with nervous } \\
\text { system, heart } \\
\text { or recurrent } \\
\text { joint } \\
\text { involvement }\end{array}$} & Ceftriaxone & $2000 \mathrm{mg}$ q24h (children: $50-75 \mathrm{mg} / \mathrm{kg} /$ day) & iv & $14-28$ \\
\hline & Cefotaxime & $\begin{array}{l}2000 \mathrm{mg} \text { tid (children: } 150-200 \mathrm{mg} / \mathrm{kg} / \text { day: } \\
\text { divided in 3-4 doses) }\end{array}$ & iv & $14-28$ \\
\hline & Penicillin G & $\begin{array}{c}\text { 3-4 mu q4h (children: } 0.2-0.4 \mathrm{mu} / \mathrm{kg} / \text { day } \\
\text { divided in } 4-6 \text { doses) }\end{array}$ & iv & $14-28$ \\
\hline \multirow[t]{5}{*}{$\mathrm{ACA}$} & Amoxicillin & $500-1000 \mathrm{mg}$ tid & po & $14-28$ \\
\hline & Doxycycline & $100 \mathrm{mg}$ bid or $200 \mathrm{mg} \mathrm{q} 24 \mathrm{~h}$ & po & $14-28$ \\
\hline & Ceftriaxone & $2000 \mathrm{mg} \mathrm{q} 24 \mathrm{~h}$ & iv & $14-28$ \\
\hline & Cefotaxime & $2000 \mathrm{mg}$ tid & iv & $14-28$ \\
\hline & Penicillin G & 3-4 mu q4h & iv & $14-28$ \\
\hline
\end{tabular}

EM - ery thema migrans, BLC - borrelial lymphocytoma cutis, ACA - atrophic chronic acrodermatitis, bid - twice a day, tid-3 times a day, po - per os (by mouth), iv - intravenously, q4h - in each $4 \mathrm{~h}, q 24 \mathrm{~h}$ - in each $24 \mathrm{~h}$ 


\section{PREVENTION}

Avoidance of tick bites is the most obvious means to prevent $B$ burgdorferi infection. If people must be outside in areas where Ixodes ticks are found, they are advised to wear protective clothing and tick repellent containing $N, N$-diethyl-m-toluamide (DEET). Frequent skin inspection and prompt removal of ticks should also decrease the risk of infection. However, data regarding the efficacy of these measures are limited. Other measures, including burning or removal of vegetation in tick-harboring areas, use of acaricides, and control of the deer population, result in upto a $94 \%$ decrease in the population of I scapularis ticks.

Studies show that, despite warnings, only $40 \%$ to $50 \%$ of adults take precautions against tick bites even when they are aware of Lyme disease ${ }^{[46]}$. Immediate removal of attached ticks can help prevent Lyme disease infection.

Therefore, complete inspection of the skin and scalp, particularly for children, is recommended after spending time outside in endemic areas.

\section{CONCLUSION}

The diagnosis should be based on objective clinical findings consistent with Lyme disease and supporting serologic tests. Most patients are cured with 2 to 4 weeks of antibiotic therapy, although the time to disease resolution may be prolonged, especially for individuals in whom therapy was delayed; irreversible tissue damage may occur. A less response to antibiotic therapy should raise concern for alternative diagnoses with other tick-borne pathogens. Treatment with NSAIDs, hydroxychloroquine, or other disease-modifying anti-rheumatic therapies usually resolve lyme arthritis within 4 to 5 years. According to a study conducted in Haryana, India there is a spread of lyme disease. A clinical pharmacist plays a major role in the prevention and early detection of the disease.

\section{REFERENCE:}

1. Cechová L, Durnová E, Sikutová S, Halouzka J, Nemec M. Characterization of spirochetal isolates from arthropods collected in South Moravia, Czech Republic, using fatty acid methyl esters analysis. J Chromatogr B Analyt Technol Biomed Life Sci 2004; 808: $249-54$.

2. Flisiak R, Prokopowicz D. Antibodies against Borrelia gariniiin diagnosis of Lyme borreliosis [Polish]. Przegl Lek 2000;57: 147-9. 
3. Halouzka J, Wilske B, Stünzner D, Sanogo YO, Hubálek Z. Isolation of Borrelia afzelii from overwintering Culex pipiensbiotype molestus mosquitoes. Infection 1999; 27: 2757.

4. Stańczak J, Racewicz M, Kubica-Biernat B, et al. Prevalence of Borrelia burgdorferi sensu lato in Ixodes ricinus ticks(Acari, Ixodidae) in different Polish woodlands. Ann AgricEnviron Med 1999; 6: 127-32.

5. de Carvalho IL, Fonseca JE, Marques JG, et al. Vasculitis-like syndrome associated with Borrelia lusitaniae infection. ClinRheumatol 2008; 27: 1587-91.

6. Derdáková $\mathrm{M}$, Lencáková $\mathrm{D}$. Association of genetic variability within the Borrelia burgdorferi sensu lato with theecology, epidemiology of Lyme borreliosis in Europe. AnnAgric Environ Med 2005; 12: 165-72.

7. Murray TS, Shapiro ED. Lyme disease. Clin Lab Med 2010;30: 311-28.

8. Eugene D. Shapiro1 and Michael A. Gerber2 Lyme Disease Clinical Infectious Diseases 2000;31:533-42

9. Wormser GP, Dattwyler RJ, Shapiro ED, et al. The clinical assessment, treatment, and prevention of Lyme disease, human granulocytic anaplasmosis, and babesiosis: clinical practice guidelines by the Infectious Diseases Society of America. Clin Infect Dis. 2006 Nov 1;43(9):1089-1134. Epub 2006 Oct 2.

10. Montiel NJ, Baumgarten JM, Sinha AA. Lyme disease, II: clinical features and treatment. Cutis. 2002;69(6):443-448.

11. Piacentino JD, Schwartz BS. Occupational risk of Lyme disease: an epidemiological review. Occup Environ Med. 2002;59(2):75-84.

12. Van Solingen RM, Evans J. Lyme disease. Curr Opin Rheumatol. 2001;13(4):293-299.

13. Centers for Disease Control and Prevention. Centers for Disease Control and Prevention Web site. http://www.cdc.gov/ncidod/dvbid/lyme. Accessed June 25, 2007.

14. Huppertz HI. Lyme disease in children. Curr Opin Rheumatol. 2001;13(5):434-440.

15. Centers for Disease Control and Prevention (CDC). Lyme disease: United States, 1996. MMWR Morb Mortal Wkly Rep. 1997;46(23):531-535.

16. Kolata G. Lyme disease is hard to catch and easy to halt, study finds. The New York Times. Wednesday, June 13, 2001:A1.

17. Poland GA. Prevention of Lyme disease: a review of the evidence. MayoClin Proc. 2001;76(7):713-724. 
18. Aschwanden C. Bitten: what every hiker needs to know about Lyme disease. Backpacker. 2006;34:74-80.

19. Steere AC. Lyme disease. N Engl J Med 1989;321:586-96.

20. Nadelman RB, Wormser GP. Lyme borreliosis. Lancet 1998;352:557-65.

21. Gerber MA, Shapiro ED, Burke GS, Parcells VJ, Bell GL, Pediatric Lyme Disease Study Group. Lyme disease in children in southeastern Connecticut. N Engl J Med 1996;335:1270-4.

22. Nadelman RB, Nowakowski J, Forseter G, et al. The clinical spectrum of early Lyme borreliosis in patients with culture-confirmed erythema migrans. Am J Med 1996; 100:502-8.

23. Steere AC, Dwyer E, Winchester R. Association of chronic Lyme arthritis with HLADR4 and HLA-DR2 alleles. N Engl J Med 1990;323:219-23.

24. Nocton JJ, Dressler F, Rutledge BJ, Rys PN, Persing DH, Steere AC. Detection of Borrelia burgdorferi DNA by polymerase chain reaction in synovial fluid from patients with Lyme arthritis. N Engl J Med 1994; 330:229-34.

25. Gross DW, Forsthuber T, Tary-Lehmann M, et al. Identification of LFA-1as a candidate autoantigen in treatment-resistant Lyme arthritis. Science1998; 281:703-6.

26. Logigian EL, Kaplan RF, Steere AC. Chronic neurologic manifestations of Lyme disease. N Engl J Med 1990;323:1438-44.

27. Logigian EL, Kaplan RF, Steere AC. Successful treatment of Lyme encephalopathy with intravenous ceftriaxone. J Infect Dis 1999;180:377-83.

28. Krause PJ, Telford SR, Spielman A, et al. Concurrent Lyme disease and babesiosis; evidence for increased severity and duration of illness. JAMA1996;275:1657-60.

29. Nadelman RB, Hororwitz HW, Hsieh TC, et al. Simultaneous human ehrlichiosis and Lyme borreliosis. N Engl J Med 1997; 337:27-30.

30. Tilly K, Rosa PA, Stewart PE. Biology of infection with Borrelia burgdorferi. Infect Dis ClinNorth Am. 2008; (22):217-234.

31. Hovius JW, van Dam AP, Fikrig E. Tick-host-pathogen interactions in Lyme borreliosis. Trends Parasitol. Sep; 2007 23(9):434-438.

32. Coleman JL, Gebbia JA, Piesman J, et al. Plasminogen is required for efficient dissemination of B. burgdorferi in ticks and for enhancement of spirochetemia in mice. Cell. Jun 27; 1997 89(7):1111-1119. 
33. Mullegger RR, McHugh G, Ruthazer R, et al. Differential expression of cytokine mRNA in skinspecimens from patients with erythema migrans or acrodermatitis chronica atrophicans. J InvestDermatol. Dec; 2000 115(6):1115-1123.

34. Fikrig E, Feng W, Aversa J, et al. Differential expression of Borrelia burgdorferi genes duringerythema migrans and Lyme arthritis. J Infect Dis. Oct; 1998 178(4):1198-1201.

35. Krause A, Brade V, Schoerner C, et al. T cell proliferation induced by Borrelia burgdorferi inpatients with Lyme borreliosis. Autologous serum required for optimum stimulation. ArthritisRheum. Apr; 1991 34(4):393-402.

36. Lyme Disease Thomas S. Murray, MD PhD1 and Associate Research Scientist of Pediatrics and Laboratory Medicine, Yale University School of Medicine Eugene D. Shapiro, MD2Professor of Pediatrics, Epidemiology and Public Health and Investigative Medicine, Yale University School of Medicine36. Steere AC. Lyme disease. N Engl J Med. 1989;321(9):586-598.

37. Kasper DL, Fauci AS, Longo DL, Braunwald E, Hauser SL, Janeson JL, eds. Harrison's Principles of Internal Medicine. 16th ed. New York, NY: McGraw-Hill Medical Publishing Division; 2005:996.

38. Walker DH. Tick-transmitted infectious diseases in the United States. Annu Rev Public Health. 1998;19:237-269.

39. Bunikis J, Barbour AG. Laboratory testing for suspected Lyme disease. Med Clin North Am. 2002;86(2):311-340.

40. Magnarelli LA, Miller JN, Anderson JF, Riviere GR. Cross-reactivity of nonspecific treponemal antibody in serologic tests for Lyme disease. J ClinMicrobiol. 1990;28(6):1276-1279.

41. Centers for Disease Control and Prevention. Centers for Disease Control and Prevention Web site. http://www.cdc.gov/ncidod/dvbid/lyme. Accessed June 25, 2007.

42. Halperin JJ, Shapiro ED, Logigian E, et al. Practice parameter: treatment of nervous system Lyme disease (an evidence-based review): report of the Quality Standards s Subcommittee of the American Academy of Neurology. Neurology 2007; 69: 91-102.

43. Wormser GP, Dattwyler RJ, Shapiro ED, et al. The clinical assessment, treatment, and prevention of lyme disease, human granulocytic anaplasmosis, and babesiosis: clinical practice guidelines by the Infectious Diseases Society of America. Clin Inf Dis 2006; 43: 1089-134. 
44. Wormser GP, Nadelman RB, Dattwyler RJ, et al. Practice guidelines for the treatment of Lyme disease. The Infectious Diseases Society of America. Clin Infect Dis 2000; 31Suppl 1: 1-14.

45. Flisiak R, Pancewicz S. Diagnostics and treatment of Lymeborreliosis. Recommendations of Polish Society of Epidemiology and Infectious Diseases [Polish]. Przegl Epidemiol 2008; 62: 193-9.

46. Poland GA. Prevention of Lyme disease: a review of the evidence. MayoClin Proc. 2001;76(7):713-724.

47. Bratton, R. L., Whiteside, J. W., Hovan, M. J., Engle, R. L., \& Edwards, F. D. (2008). Diagnosis and treatment of lyme disease. Mayo Clinic Proceedings, 83(5), 566571.

48. Diagnosis, Treatment, and Prevention of Lyme Disease, Human Granulocytic Anaplasmosis, and Babesiosis A Review Edgar Sanchez, MD; Edouard Vannier, PhD; Gary P. Wormser, MD; Linden T. Hu, MD

49. Lyme Disease Eugene D. Shapiro1 and Michael A. Gerber Of ticks, mice and men: understanding the dual-host lifestyle of Lyme disease spirochaetes Justin D. Radolf, Melissa J. Caimano, Brian Stevenson \& Linden T. Hu

50. Reprinted from Trends in Microbiology, Vol. 21, No. 8, Coburn, J., Leong, J. and Chaconas, G., Illuminating the roles of the Borrelia burgdorferi adhesins, Pages 372-379, 51. Lyme Disease, Kelley and Firestein's Textbook of Rheumatology (Tenth Edition)Volume 2, 2017, Pages 1891-1904. 